\title{
Overdiagnosis and overtreatment of Lyme neuroborreliosis are preventable
}

\author{
Avinash Prasad, Douglas Sankar
}

\begin{abstract}
Summary
The problems of diagnosis and treatment of Lyme neuroborreliosis can be minimised by strictly following the clinical diagnostic criteria, and understanding the pitfalls of laboratory tests. The diagnosis is based solely on objective clinical findings, with serologic test results used only to confirm the diagnosis. It must be underscored that serologic testing, when ordered without regard for clinical presentation (ie, used as a screen), may be misleading due to its extremely low positive predictive value. Enzyme-linked immunosorbent assay should always be confirmed by Western blot. The cerebrospinal fluid Borrelia burgdorferi antibody index is more meaningful than simple titres of specific antibody. Polymerase chain reaction is still a research tool and should not be utilised without clinical correlation. All serologic tests and polymerase chain reaction may remain positive long after successful treatment. Overdiagnosis and overtreatment can be minimised by following these guidelines.
\end{abstract}

Keywords: Lyme disease; neuroborreliosis; Borrelia burgdorferi

Department of Neurology, New York University Medical Center, New York, NY 10016, USA

A Prasad

D Sankar

Correspondence to Avinash Prasad MD, 212 Copeley Road \#3, Charlottesville, VA 22903, USA

Accepted 7 April 1999
Lyme disease has become a leading vector-borne infectious disease all over the world, with $10-40 \%$ of patients eventually developing Lyme neuroborreliosis. ${ }^{1}$ This clinical entity is another great mimicker, like tuberculosis and syphilis, as its clinical manifestations are protean. The high prevalence and mimicker status of Lyme neuroborreliosis increases the physician's responsibility for precise diagnosis and treatment.

In spite of advances in the diagnostic armamentarium, the diagnosis of Lyme disease and Lyme neuroborreliosis remains problematic. In one study only a third of patients referred to a Lyme disease clinic were found to have Lyme disease, either active or by history. ${ }^{2}$ Diseases such as depression and cancer may be overlooked, if guidelines for diagnosis and treatment of Lyme neuroborreliosis are not followed carefully. In this paper, the importance of strictly following the criteria for clinical diagnosis is emphasised, and the pitfalls of the diagnostic methods are discussed.

\section{Clinical features}

A clinical consensus currently divides Lyme neuroborreliosis into early disseminated and late stages. Both the central nervous system (CNS) and peripheral nervous system (PNS), along with the blood vessels and meningeal coverings, may be involved in either stage. However, the pattern of nervous system involvement is different (table 1). This classification is helpful in understanding the correlation of the clinical spectrum of Lyme neuroborreliosis with serologic tests and treatment. Subtle differences in the clinical presentation of early and late Lyme neuroborreliosis patients in Europe and North America are related to the different Borrelia burgdorferi $(\mathrm{Bb})$ genospecies on the two continents.

EARLY DISSEMINATED LYME NEUROBORRELIOSIS

The classic triad of this stage, also known as meningoradiculoneuropathy, includes aseptic meningitis, cranial neuropathy, and painful radiculoneuritis which may occur alone or in combination. Acute encephalomyelitis is seen less frequently. Neurologic signs and symptoms develop from weeks to months after inoculation. The onset is acute or subacute. Accompanying erythema migrans and systemic signs and symptoms characteristic of early disseminated Lyme neuroborreliosis, may be present in up to two-thirds of cases. Neurologic involvement can occur without erythema migrans, however, and can be the first manifestation of the disease. ${ }^{3}$ Only $40 \%$ of patients recall tick bite. The absence of a history of travel to or residence in an endemic area tends to warn the clinician against the diagnosis of Lyme neuroborreliosis.

Table 1 Characteristic features of early and late Lyme neuroborreliosis

\begin{tabular}{|c|c|c|}
\hline & Early disseminated $L N B$ & Late $L N B$ \\
\hline Onset & Acute & Insidious \\
\hline \multirow[t]{2}{*}{ CNS } & Lymphocytic meningitis & Subtle chronic encephalopathy \\
\hline & $\begin{array}{l}\text { Acute encephalomyelitis (focal hemispheric, } \\
\text { basal ganglion, brainstem, cerebellum and } \\
\text { spinal cord features) }\end{array}$ & Chronic encephalomyelitis \\
\hline \multirow[t]{2}{*}{ PNS } & $\begin{array}{l}\text { Acute radiculoneuropathy (mononeuritis } \\
\text { multiplex) }\end{array}$ & $\begin{array}{l}\text { Chronic radiculoneuropathy } \\
\text { (mononeuritis multiplex) }\end{array}$ \\
\hline & Cranial neuropathy & \\
\hline CSF pleocytosis & $75-85 \%$ & $\begin{array}{l}5 \% \text { in CNS LNB, absent in PNS LNB } \\
50 \% \text { in CNS LNB }\end{array}$ \\
\hline Anti-Bb Ab & $80-90 \%$ & Rarely present in PNS LNB \\
\hline Treatment & Excellent & Slow and variable \\
\hline
\end{tabular}

CNS = central nervous system, PNS = peripheral nervous system, LNB = Lyme neuroborreliosis, Anti-Bb $\mathrm{Ab}=$ anti-Borrelia burgdorferi antibody 
Meningitis is the single most common presentation in early disseminated Lyme neuroborreliosis. Headache is the main complaint. Fever and meningismus are usually mild or absent. Therefore, a high index of suspicion is often required for the diagnosis. Somnolence, irritability, impaired memory, depressed mood, and behavioural changes are seen in half of the patients and may be interpreted as features of meningoencephalitis, but are difficult to separate from the effects of meningitis. The differential diagnosis includes other causes of aseptic meningitis. Examination of cerebrospinal fluid (CSF) shows a mononuclear pleocytosis, with elevated protein and normal glucose levels. CSF anti-Bb antibody (usually IgG or IgA) is present in $80-90 \%$ of patients, whereas CSF polymerase chain reaction (PCR) is positive in less than half of the patients.

About two-thirds of patients with early disseminated Lyme neuroborreliosis have cranial neuropathies which can occur without other neurologic or systemic features and without preceding erythema migrans. ${ }^{4}$ The facial nerve is involved most commonly, although any of the cranial nerves may be involved. Remarkably, bilateral facial palsy is seen in approximately one-third of such cases. The differential diagnosis of bilateral facial palsy includes Guillain-Barre syndrome, acquired immunodeficiency syndrome (AIDS), various other causes of meningitis, and Tangier's disease.

Radiculoneuropathy develops in about one-third of patients with early Lyme neuroborreliosis. Severe radicular pain, paraesthesia and hyperaesthesia are the most common complaints. Motor and sensory deficits are typically asymmetric and multifocal: plexus, nerve roots and nerves may be involved, either singly or in combination. Uncommon presentations include paralysis of the diaphragm or abdominal muscles. ${ }^{2}$ Rarely, patients may present with Guillain-Barre syndrome and CSF pleocytosis. ${ }^{5}$ The differential diagnosis includes diseases with neuropathy and/or CSF pleocytosis such as disc prolapse, diabetic proximal neuropathy, vasculitic neuropathy, cytomegalovirus radiculopathy, neurosarcoidosis and zoster sine herpete.

In meningoradiculoneuropathy, CSF shows features of meningitis. Headache, fever, facial palsy, truncal sensory/motor loss, and features of mononeuritis multiplex support the diagnosis of Lyme aetiology.

Acute encephalomyelitis, ie, brain and spinal cord parenchymal involvement, occurs in $10-20 \%$ cases of early disseminated Lyme neuroborreliosis in Europe, but rarely in North America. Diffuse involvement of the cerebral hemispheres may manifest as hallucinations, agitation, hypersomnolence, confusion, coma and seizures. Focal signs referable to cerebral hemispheres, basal ganglion, brainstem, cerebellum and spinal cord may be present. Virtually all patients have CSF lymphocytic pleocytosis.

LATE LYME NEUROBORRELIOSIS

Neurological manifestations usually develop months or years after the inoculation and may present as a syndrome with typical CNS and PNS abnormalities.

\section{CNS abnormalities}

The clinical presentation varies between North America and Europe. Encephalopathy is more common in North America, while encephalomyelitis is much more frequent in Europe. Encephalopathy is usually mild to moderate and may persist for years, if untreated. Complaints are usually vague: impaired memory and concentration, insomnia and hypersomnolence. Mild psychiatric disturbance in the form of depression, irritability and paranoia may be present. Chronic fatigue is a common accompaniment, but it never occurs alone. ${ }^{67}$ Objective neurologic findings are lacking. The differential diagnosis includes chronic fatigue syndrome, fibromyalgia, post-Lyme syndrome, depression, normal aging, early Alzheimer's disease and neurosyphilis. The clue to the diagnosis is a history of tick exposure or early cardinal clinical features of Lyme disease. Logigian, in a series of about 100 Lyme encephalopathy patients, found only one who did not have well recognised manifestations of early Lyme disease. ${ }^{8}$

Serum immunoreactivity by enzyme-linked immunosorbent assay (ELISA) and Western blot is nearly universal. However, seroreactivity only indicates exposure to the organism and does not establish that $\mathrm{Bb}$ is the cause of encephalopathy. CSF pleocytosis is present in 5\% and protein content is increased in $20-45 \%$ of cases. Specific anti-Bb antibody is present in the CSF in half of cases. CSF PCR may be positive.

Overall, the patients with symptoms of encephalopathy fall into two groups. The first group of patients suffer from active Lyme neuroborreliosis. They usually show impaired performance on objective memory tests. CSF is abnormal in most cases, implying a neurologic basis. ${ }^{9}$ The symptoms of encephalopathy are usually reversible with antibiotic treatment. The second group comprises patients in whom the symptoms are possibly due to the psychological stress of 
Lyme disease,${ }^{10}$ depression, ${ }^{11}$ toxic-metabolic effect of a systemic infection, ${ }^{12}$ or an immune reaction. ${ }^{13}$ Examination of CSF in such cases does not usually reveal abnormalities. Formalised neuropsychological tests can be of great use in distinguishing between these two groups of patients. Although the pattern of abnormality seen in patients with Lyme encephalopathy is not unique, it is sufficiently different from patterns that occur with depression, anxiety, or Alzheimer's disease to differentiate Lyme encephalopathy from other causes of concentration and memory impairment. ${ }^{14}$ After antibiotic treatment, the neuropsychologic abnormalities have been reported to improve significantly.

Most cases of chronic encephalomyelitis have been reported from Europe, although well-documented North American cases have also been described. ${ }^{15}$ Neurologic signs may be multilevel, reflecting involvement of brain, spinal cord, and cranial and peripheral nerves. Insidious onset and progressive worsening over months to years distinguishes this from similar features seen in early Lyme neuroborreliosis. In most cases, the clinical features of late Lyme neuroborreliosis do not resolve spontaneously. Variable improvement may be noted after antibiotic treatment.

\section{PNS abnormalities}

About one-third of patients with late Lyme neuroborreliosis suffer from mild chronic radiculoneuropathy. This is different from the meningoradiculoneuropathy seen in the early disease. It causes less striking signs and symptoms and does not improve spontaneously. Motor involvement is rare. Sensory symptoms are usually asymmetric and patchy. Radicular pain in the limbs or trunk is less common. Neurologic examination is usually normal. The CSF of patients with neuropathy alone is rarely abnormal. However, with concomitant encephalopathy, increased CSF protein content and intrathecal production of anti-Bb antibody may be seen. A more severe peripheral neuropathy with unique skin changes, known as acrodermatitis chronica atrophicans, is common in Europe. This is manifested as bluish-red oedematous cutaneous lesions on the feet at onset. Atrophy and wrinkling of the involved skin occurs later.

\section{Laboratory diagnosis}

Laboratory support is necessary only to confirm the diagnosis (table 2). The diagnosis should never be made solely on the basis of serologic tests. It is prudent not to order serologic tests for patients with vague symptoms such as chronic fatigue in isolation without any cognitive symptoms or other Lyme-related finding.

\section{CSF EXAMINATION}

Changes in CSF include: lymphocytic pleocytosis of up to 3750 cells $/ \mathrm{mm}^{3}$ (usually $100-170$ ), protein $100-300 \mathrm{mg} / \mathrm{dl}$ (or even up to $1300 \mathrm{mg} / \mathrm{dl}$ in cases of longer duration), slightly low glucose (only in cases of longer duration), and the presence of oligoclonal bands of IgG in about $90 \%$ of cases. While abnormal $\mathrm{CSF}$ in a seropositive person helps confirm that a neurologic syndrome is due to $\mathrm{Bb}$, normal CSF should not be used to exclude Lyme neuroborreliosis. Documentation of the extent of CSF abnormalities provide a baseline from which the subjective improvement may be confirmed by objective improvement in the CSF. The abnormalities should show improvement on repeat examination, although immune markers as well as increased protein may remain present in the CSF for many years.

Isolation of the organism is the gold standard test for the diagnosis for the majority of the bacterial diseases. $\mathrm{Bb}$ is difficult to culture from biological specimens due to its fastidious growth habits and low number. Since a positive CSF culture is seen in less than $10 \%$ of Lyme neuroborreliosis cases, isolation of $\mathrm{Bb}$ is less useful diagnostically. ${ }^{16}$

Table 2 Current status of laboratory procedures for diagnosis for Lyme neuroborreliosis

\begin{tabular}{|c|c|c|}
\hline Laboratory tests & Advantages & Limitations \\
\hline $\begin{array}{l}\text { CSF culture } \\
\text { Serum ELISA }\end{array}$ & Gold standard & $\begin{array}{l}\text { Low yield procedure } \\
\text { False +ve/ false-ve common } \\
\text { Interlaboratory variability + }\end{array}$ \\
\hline 2 step Serum ELISA \& Western blot & Highly sensitive and specific for systemic LD & $\begin{array}{l}\text { Occasional false +ve } \\
\text { Western blot less objective }\end{array}$ \\
\hline $\begin{array}{l}\text { Intrathecal anti-Bb } \mathrm{Ab} \text { index } \\
\mathrm{PCR}\end{array}$ & $\begin{array}{l}\text { Highly specific for LNB } \\
\text { Sensitivity moderate to high }\end{array}$ & $\begin{array}{l}\text { False -ve in late CNS; absent in late PNS LNB; occasional false +ve } \\
\text { Research tool at present; false +ve if contamination }\end{array}$ \\
\hline
\end{tabular}

$\mathrm{CSF}=$ cerebrospinal fluid, anti-Bb Ab $=$ anti-Borrelia burgdorferi antibody, $\mathrm{PCR}=$ polymerase chain reaction, $\mathrm{CNS}=$ central nervous system, $\mathrm{PNS}=$ peripheral nervous system, $\mathrm{LD}=$ Lyme disease, $\mathrm{LNB}=$ Lyme neuroborreliosis 
SERUM ANTIBODY TESTS

Serum antibody tests are useful for the diagnosis of Lyme disease. In Lyme neuroborreliosis, they play an important role in detecting not only previous exposure to $\mathrm{Bb}$, but also the systemic activity of the organism. ELISA has replaced indirect immunofluorescent assay as the serum antibody test of choice because it is more objective. Its important drawback is false positive results, which occur in diseases such as other spirochetal diseases, tuberculous meningitis, bacterial endocarditis, rheumatoid arthritis, systemic sclerosis, infectious mononucleosis, Rocky Mountain spotted fever and AIDS.

An early negative serologic result does not rule out the disease, as a second specimen taken 3-4 weeks later may be positive. Failure of the seroconversion of the second specimen may be expected if antibiotic treatment were started promptly, based on the clinical diagnosis. In those cases when the clinical diagnosis is less certain and the decision is made not to treat the patient, a negative second specimen argues against the diagnosis of Lyme disease/Lyme neuroborreliosis.

Positive serologic tests in early Lyme disease are usually low-level positives. High titer in the early stage of the disease indicates past or recurrent infection. A positive serologic test, regardless of titer, indicates past exposure to $\mathrm{Bb}$ and does not reflect disease activity. Therefore, the presence of serum reactivity alone is not sufficient to diagnose Lyme neuroborreliosis.

A high level positive serology is seen in the majority of chronic Lyme disease/Lyme neuroborreliosis cases. However, negative, low level or decreasing Bb titers can occur in untreated chronic Lyme disease cases due to impaired immune response. ${ }^{17}$ Patients presenting with possible late Lyme neuroborreliosis and a low reactive or borderline $\mathrm{Bb}$ titer should be thoroughly examined for other diseases.

Western blot or immunoblot is considered more specific than ELISA for diagnosing Lyme disease/Lyme neuroborreliosis. It is less often falsely positive than ELISA, as long as the criteria of a sufficient number of antibody bands required for the diagnosis, is satisfied. However, there are a few inherent problems with this test. Unlike HIV Western blot, Lyme Western blot is not a confirmatory test because of the presence of common and cross-reacting bacterial antigens expressed by different $\mathrm{Bb}$ genospecies. Reading of the band intensity is subjective and needs a lot of experience. If the treatment of Lyme neuroborreliosis has been initiated early on the basis of clinical criteria, the test result may be negative.

To improve the diagnostic efficacy in Lyme neuroborreliosis, the US Center for Disease Control recommends a two-step serologic evaluation. $\mathrm{Bb}$ Flagella-based ELISA (FLA-ELISA), the first step, is a screening test because of the presence of flagellin epitopes in numerous other bacteria. IgM and $\operatorname{IgG}$ Western blot, the second step, is performed only for samples scored positive or equivocal by the ELISA. The Western IgG blot is considered positive if five of the following 10 bands are present:18, 23, 28, 30, 39, 41, 45, 60, 66 and $93 \mathrm{kDa}$. Western IgM blot is considered positive if two of the following bands are present: 23, 39, $41 \mathrm{kDa} .{ }^{18}$ The sensitivity of the combined two-step ELISA and Western blot was $100 \%(17 / 17)$ in a recent study in early and late Lyme neuroborreliosis. The specificity was $100 \%$ (0/113) for healthy blood donors and 90\% (11/111) for persons with autoimmune disorders. ${ }^{19}$ More studies are needed to confirm these results.

CSF ANTIBODY TEST

$\mathrm{CSF}$ anti-Bb antibody index is currently the best indicator of Lyme neuroborreliosis. It measures brain compartment synthesis of spirochete-specific antibodies and is much more meaningful than total specific antibody in CSF, which reflects passive diffusion of blood antibody. The technique normalises CSF and serum to the same IgG concentration, then runs the paired samples for anti-Bb antibodies by routine ELISA. Local CSF antibody production is implied when the CSF anti-Bb antibody index (CSF optical density / serum optical density) is greater than one.

Intrathecal anti-Bb antibody production is helpful when positive, because it strongly suggests CNS involvement by the spirochete. In spite of high specificity, false positive intrathecal anti- $\mathrm{Bb}$ antibody production was noted in three out of 77 patients in one series. ${ }^{20}$ Also, a negative result does not rule out Lyme neuroborreliosis. Intrathecal anti-Bb antibody was positive in $92 \%$ of patients with meningitis, and $42 \%$ of patients with late CNS Lyme neuroborreliosis, but was absent in patients with late PNS Lyme neuroborreliosis in one series. ${ }^{21}$

CSF-PCR

The combination of a variable serologic response and difficult isolation has made detection of spirochetal genomic material an area of interest. PCR-based methods can detect less than $10 \mathrm{Bb}$ organisms in the biologic samples and such 


Major pitfalls in the diagnosis
of Lyme neuroborreliosis
Patient interpreted to have active or
persistent Lyme neuroborreliosis because of:
- positive Lyme serology at the onset of
disease
- persistently positive Lyme serology
even after adequate therapy
- persistently positive PCR even after
adequate therapy
- persistent vague symptoms after
adequate treatment (post-Lyme
disease syndrome)
Patient interpreted not to have Lyme
neuroborreliosis because of:
- no history of tick-bite
- negative Lyme serology
- negative CSF
negative PCR

detection is highly specific. However, PCR has two important drawbacks. First, the extreme sensitivity of PCR can result in false positive result due to contamination. This problem can be controlled in a research laboratory by careful attention to controls and to laboratory technique. Second, the presence of PCR-detectable organisms does not prove the presence of clinically active disease because the organisms may be dead or alive. ${ }^{22}$

In one study, ${ }^{22}$ nested pairs of oligonucleotide primers were designed to recognise the C-terminal region of $\mathrm{Bb}$ outer surface protein $\mathrm{A}$ (OspA). PCR detected $\mathrm{Bb}$ OspA DNA in 10 of 11 patients with Lyme encephalopathy, 28 of 37 patients with inflammatory Lyme neuroborreliosis, seven of seven seronegative patients with Lyme-compatible disorders, and none of 23 patient controls. In addition, PCR results predicted clinical results accurately in eight patients in whom CSF was tested before and after parenteral antibiotics. ${ }^{22}$ In other studies, however, PCR was found to have methodological problems and was positive in only $25-50 \%$ of cases. ${ }^{23}{ }^{24}$ More studies are needed to evaluate its sensitivity and specificity before it becomes a routine test. Nevertheless, PCR may be used to corroborate the diagnosis in equivocal cases.

\section{Diagnosis}

The diagnosis of definite Lyme neuroborreliosis can be made when there is a history of exposure to appropriate ticks in an area endemic for Lyme disease, a compatible neurologic abnormality without other cause, and at least one of the following:

- history of well-documented erythema migrans

- history or presence of lymphocytoma or ACA

- seroconversion or four-fold rise in titer of anti-Bb antibodies in paired serum specimens

- intrathecal CSF anti-Bb antibody production

- other organ system involvement typical of Lyme disease with raised titers of specific serum antibody ${ }^{25}$

- culture, histologic or PCR proof of presence of $\mathrm{Bb}^{26}$

Until a 'gold standard' diagnostic test is established, it is reasonable to accept a less restrictive criteria for the diagnosis of Lyme neuroborreliosis, for example, a compatible neurologic abnormality without other cause, and either serum immunoreactivity to $\mathrm{Bb}$ with no demonstrable rise in the antibodies, or tick bite or travel or residence in an endemic area. ${ }^{25}$ Failure to treat these probable or possible cases in time, may have far reaching consequences. ${ }^{26}$ Other diagnoses should always be considered in these cases before antibiotic treatment is prescribed. Major pitfalls in the diagnosis of Lyme neuroborreliosis are summarised in the box.

\section{Treatment}

An algorithm for the diagnosis and treatment is provided in the figure. Lyme neuroborreliosis is not a medical emergency in the majority of cases. Therefore, a thorough evaluation of the patient must be done before making major management decisions.

Controversy exists regarding management of patients with mild confusion/ memory changes, absent Lyme-related objective clinical findings, absent CSF pleocytosis and absent serum or CSF $\mathrm{Bb}$ antibodies and in whom late Lyme neuroborreliosis is suspected. Unequivocal clinical guidelines are lacking. Most experts believe that this clinical situation is distinctly unusual, ${ }^{6}$ and suggest that repeating the tests in a different laboratory or Bb DNA CSF $\mathrm{PCR}^{6}$ may be helpful in some instances. Halperin et al suggest that empirical oral antibiotic therapy may be effective in such cases. ${ }^{26}$ On the other hand, treating such cases with antimicrobial agents is considered questionable by some. ${ }^{6}$

Invasion of the brain parenchyma by spirochete-like organisms has been demonstrated by immunochemistry, and with great difficulty by silver impregnation technique in humans. ${ }^{27}$ On this premise, it is hypothesized that Lyme meningitis is a meningoencephalitis. Therefore, when designing a treatment regimen, one must pay attention not only to sensitivity of the organism but also to the ability of the regimen to provide adequate CSF and, if possible, brain levels.

Once neurologic abnormalities develop, parenteral treatment is usually required. Patients with early Lyme neuroborreliosis improve with parenteral antibiotic therapy, although they have also been known to recover without any therapy. Antibiotics certainly lead to a faster resolution of symptoms. The usual duration of therapy is about $2-4$ weeks. Meningitis resolves within days to weeks; other features may take months to improve. An exception to the use of parenteral 
Figure Algorithm for the diagnosis and therapy of Lyme neuroborreliosis. CSF= cerebrospinal fluid; Anti-BbAb = Borrelia burgdorferi antibody; serum E\&W = serum 2-step ELISA and Western blot; LNB = Lyme neuroborreliosis, LD = Lyme disease

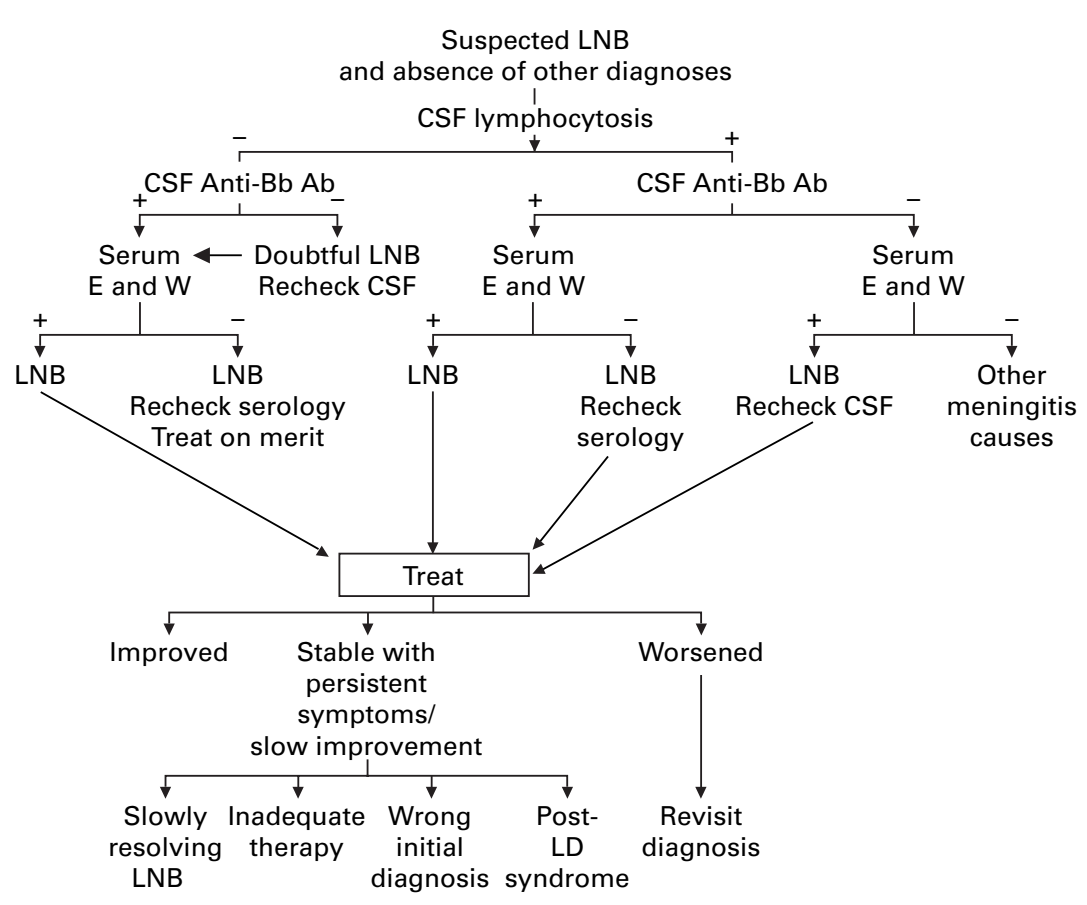

therapy is the patient with uncomplicated facial palsy without CSF abnormalities for whom oral antibiotic therapy for $2-4$ weeks is adequate (table 3 ).

The natural history of untreated late disseminated Lyme neuroborreliosis is less well understood. The majority of patients improve with antibiotic therapy. Recovery is slow and often incomplete and may take nearly a year. The optimal duration of therapy is unknown; because as many as $10 \%$ of patients who improve initially following 10-14 days therapy may relapse, intravenous antibiotics are often prescribed for 4 weeks. ${ }^{10}$ In one multicentre trial, there was no significant difference in the results of 2 or 4 weeks therapy with intravenous ceftriaxone for late disseminated Lyme neuroborreliosis. ${ }^{28}$ Published studies do not support the use of parenteral antibiotic therapy for longer than $4-6$ weeks ${ }^{29}$ because $\mathrm{Bb}$ resistance to penicillin and ceftrixone has not yet been reported. Further, the persistence of antibody titers and PCR positivity do not always mean persistent infection. ${ }^{30}$ Follow-up serologic testing in the patient who is asymptomatic or who is slowly improving should be discouraged.

All neurologic abnormalities can be treated with intravenous penicillin (20 million units/day) or ceftriaxone ( $2 \mathrm{~g}$ once a day). In one study, ceftriaxone proved to be superior to penicillin ${ }^{31}$ and it does have the advantage of once-a-day dosing. Another study showed similar results. ${ }^{32}$ Cefotaxime is an acceptable alternative. There is evidence that oral or intravenous doxycycline may also be effective. It may be useful in Lyme neuroborreliosis patients who are allergic to penicillin and cephalosporin. The role of corticosteroid in the treatment of Lyme neuroborreliosis has not been clearly established. In a long-term follow-up series, patients treated with antibiotic and steroid tended to recover faster than those with antibiotic alone. ${ }^{33}$

Long-term parenteral antibiotic therapy is costly and exposes patients to a variety of complications, including some that are potentially life-threatening. ${ }^{34}$ Some clinicians continue to treat Lyme neuroborreliosis patients in the absence of a clinical response, with the presumption of a 'resistant' or 'dormant' infection. After careful analysis of patients referred to a specialised centre, it was found that persistence of symptoms may be due to one of the following causes:

- slowly resolving Lyme neuroborreliosis

- irreversible tissue damage

- true persisting disease due to inadequate therapy

- post-Lyme disease syndrome

- initial misdiagnosis. ${ }^{35}$

Refractory and recurrent Lyme disease is rare. Thus, unless the patient is getting worse, the clinician should delay retreatment until enough time has elapsed for a full clinical recovery from initial treatment. ${ }^{36}$ 
Table 3 Antimicrobial therapy of Lyme neuroborreliosis

\begin{tabular}{lll}
\hline Condition & Recommended therapy & Alternative therapy \\
\hline $\begin{array}{l}\text { Meningitis, radiculoneuritis, } \\
\text { Encephalitis, polyneuropathy, encephalomyelitis. } \\
\text { Cranial neuritis (normal CSF) }\end{array}$ & $\begin{array}{l}\text { ceftriaxone } \times 2-4 \text { weeks } \\
\text { ceftriaxone } \times 2-4 \text { weeks } \\
\text { doxycycline } \times 2-4 \text { weeks }\end{array}$ & $\begin{array}{l}\text { penicillin, cefotaxime } \\
\text { penicillin, cefotaxime } \\
\text { amoxicillin, ceftriaxone }\end{array}$ \\
\hline
\end{tabular}

Complaints of headaches, arthralgias, fatigue, malaise, mild cognitive abnormalities, and sleep disturbances even after adequate antibiotic therapy is known as 'post-Lyme disease syndrome'. ${ }^{35}$ This phenomenon is not unique to Lyme disease. Similar symptoms have been noted after influenza, hepatitis and infectious mononucleosis. Controversy exists regarding its pathogenesis. A protracted immune activation by the bacterium is implicated. Symptomatic treatment with ibuprofen or amitriptyline, and exercise may be helpful.

Patients with new onset fatigue, depression and a positive Lyme serology should be treated on the merits of history (tick bite, joint swelling, skin manifestations). If cognitive symptoms are present, spinal tap is advisable. Treatment is necessary if CSF is abnormal. Extraordinary emphasis placed on vague symptoms like depression and fatigue may lead to errors in diagnosis.

1 Pachner AR, Steere AC. The triad of neurologic manifestations of Lyme disease: meningitis, cranial neuritis and radiculoneuritis. Neurology 1985;35:47-53.

2 Sigal LH. Experience with the first one hundred patients referred to a Lyme disease referra center. Am f Med 1990;88:577-83.

3 Reik L. Lyme disease and the nervous system. New York: Thieme Medical Publishers, 1991; pp 57-61

4 Clark JR, Carlson RD, Sasaki CT, Pachner AR, Steere AC. Facial paralysis in Lyme disease. Laryngoscope 1985;95:1341-5.

5 Sterman AB, Nelson S, Barclay P. Demyelinating neuropathy accompanying Lyme disease. Neurology 1982;32:1302-5.

6 Pachner A R. Lyme neuroborreliosis. In: Johnson RT, Griffin JW, eds. Current therapy in neurologic disease. St Louis: Mosby, 1997; pp 140-6.

7 Coyle P K. Lyme disease. In: Feldmann E, ed. Current diagnosis in neurology. St Louis: Mosby, 1994; pp 110-4.

8 Logigian E L. Neurologic manifestations of Lyme disease. In: Rahn QW, Evans J, eds. Lym disease. Philadelphia: ACP, 1998; pp 89-106.

9 Kaplan F R, Jones-Woodward L. Lyme encephalopathy: a neuropsychological perspective. Semin Neurol 1997;17:31-7.

10 Logigian EL, Kaplan RF, Steere AC. Chronic neurologic manifestations of Lyme disease. $N$ Engl F Med 1990;323:1438-44.

11 Finkel MJ, Halperin JJ. Nervous system Lyme neuroborreliosis revisited. Arch Neurol 1992;49: $102-7$.

12 Halperin JJ, Krupp LB, Golightly MG, Volkman DJ. Lyme borreliosis-associated encephalopathy. Neurology 1990;40:1340-3.

13 Krupp LB. Lyme disease. In: Samuels MA Feske S, eds. Office practice of neurology. London: Feske S, eds. Office practice of neurology. Lonc
Churchill-Livingstone, 1996; pp 383-7.

14 Sigal LH. Long-term consequences of Lyme disease. In: Rahn QW, Evans J, eds. Lyme disease. Philadelphia: ACP, 1998; pp 137-53.
15 Kohler J, Kern U, Kasper J, Rhese-Kupper B, Thoden U. Chronic central nervous system Thoden U. Chronic central nervous system
involvement in Lyme borreliosis. Neurology involvement in

16 Karlsson M, Hovind-Hougen K, Svenungsson $\mathrm{B}$, Stiernstedt G. Cultivation and characterization of spirochetes from cerebrospinal fluid of patients with Lyme borreliosis. F Clin Microbiol 1990;28:473-9

17 Barbour AG. Laboratory aspects of Lyme borreliosis. Clin Microbiol Rev 1988;1:399- 414.

18 Dressler F, Whalen JA, Reinhardt BN, Steere A. Western blotting in the serodiagnosis of Lyme disease. F Infect Dis 1993;167:392-400

19 Johnson BJB, Robbins KE, Bailey RE, et al. Serodiagnosis of Lyme disease: Accuracy of a two-step approach using a flagella-based ELISA and immunoblotting. F Infect Dis 1996;174:34653.

20 Treib J, Woessner R, Dobler G, Fernandez A Hozler G, Schimrigk K. Clinical value of Hozler G, Schimrigk K. Clinical value of
specific intrathecal production of antibodies. specific intrathecal product
Acta virol 1997;41:27-30.

21 Steere AC, Berardi VP, Weeks KE, Logigian EL, Ackermann R. Evaluation of the intrathecal antibody response to Borrelia burgdorferi as a diagnostic test for Lyme neuroborreliosis. $\mathcal{F}$ Infect Dis 1990;161:1203-9.

22 Keller TL, Halperin JJ, Whitman M. PCR detection of Borrelia burgdorferi DNA in cerebrospinal fluid of Lyme neuroborreliosis patients. Neurology 1992;42:32-41.

23 Pachner AR, Delaney E. The polymerase chain reaction in the diagnosis of Lyme neuroborreliosis. Ann Neurol 1993;34:544-50.

24 Nocton JJ, Bloom BJ, Rutledge BJ, Logigian $\mathrm{EL}$, Schmid $\mathrm{CH}$, Steere AC. Detection of Borrelia burgdorferi DNA by polymerase chain reaction in cerebrospinal fluid in Lyme neureaction in cerebrospinal fluid in Lyme

25 Reik L. Neurologic aspects of North American Lyme disease. In : Coyle P K ed. Lyme disease. St Louis: Mosby Year Book 1993; pp101-12.
26 Halperin JJ, Logigian EL, Finkel MF, Pearl RA. Practice parameters for the diagnosis of patients with nervous system Lyme borreliosis (Lyme disease). Neurology 1996;46:619-27.

27 Pachner AR, Duray P, Steere AC. Central nervous system manifestations of Lyme disease. Arch Neurol 1989;46:790-5.

28 Dattwyler RJ, Luft BJ, Maladorno D, et al. Treatment of late Lyme disease - a comparison of 2 weeks vs 4 weeks of ceftriaxone. VII International Congress on Lyme Borreliosis. San Francisco, June, 1996.

29 Wormser GP. Treatment and prevention of Lyme disease, with emphasis on antimicrobial therapy for neuroborreliosis and vaccination. Semin Neurol 1997;17:45-52.

30 Feder HM Jr, Gerber MA, Luger SW, Ryan SW. Persistence of serum antibodies to Borrelia burgdorferi in patients treated for Lyme disease. Clin Infect Dis 1992:15;788-92.

31 Dattwyler RJ, Halperin JJ, Volkman DJ, Luft BJ. Treatment of late Lyme borreliosis - randomized comparison of ceftriaxone and penicillin. Lancet 1988;1:1191-4.

32 Mulleger RR, Millner MM, Stanek, Spork KD. Penicillin $G$ and ceftriaxone in the treatment of neuroborreliosis in children - a prospective study. Infection 1991;19:279-83.

33 Kruger H, Kohlhepp W, Konig S. Follow-up of antibiotically treated and untreated euroborreliosis. Acta Neurol Scand 1990;82:59-67.

34 Nadelman RB, Arlen Z, Wormser GP. Life threatening complications of empiric ceftriaxone for 'seronegative' Lyme disease. South Med f 1991;84:1263-5.

35 Sigal LH. Persisting symptoms. In: Coyle P K ed. Lyme Disease. St. Louis: Mosby Year Book 1993; pp 187-91.

36 Sigal LH. Current recommendations for the treatment of Lyme disease. Drugs 1992;43:68399. 